\title{
Superhydrophobic and Oleophilic Polymeric Open Cellular Foams for Efficient Oil/Water Separation
}

\author{
Yifeng Huang and Chul B Park* \\ Microcellular Plastics Manufacturing Laboratory (MPML), \\ Department of Mechanical and Industrial Engineering, \\ University of Toronto, Canada \\ park@mie.utoronto.ca
}

\begin{abstract}
Oil spillage and discharge of organic solvents have caused a wide range of short- and long-term damage to the ecosystems of rivers, lakes, and oceans. Meanwhile, the oil-contaminated wastewater coming from different sources including oil production, pharmaceutical, and chemical industries is also detrimental to the environment. Among all techniques used to tackle this issue, the polymeric open-cell foams with super-hydrophobicity and oleophilicity are gaining increased attention for oil/water separation applications, due to their fascinating porous structure and surface properties. However, developing sustainable and environmentally friendly foam-based absorbent materials with enhanced separation efficiency remains a challenging task. This speech at first briefly reviews the chemical and engineering methods with a major focus on plastic foaming technology. Surface modification strategies on those open-cell foams to enhance the separation efficiency are also included. By investigating the effects of pore structures, void fraction, and surface properties on the separation performance, we attempt to establish the relationship between the microstructure and the performance of oil/water separation. Also, we provide a deep analysis of the dynamics of capillary oil flow and the water resistance of the open-cell foams with super-wetting properties. Moreover, two types of pilot-scale oil/water separation devices based on open-cell foam absorbents are described. Finally, we point out the challenges, as well as future perspectives and recommendations aiming at further improvement of the sustainability, cost-effectiveness, practical and economic feasibility, and the scalability of current oil/water separation technologies based on foam-based advanced absorbent materials.
\end{abstract}

